Pacific Journal of Mathematics

作 


\title{
ON CONTINUOUS IMAGE AVERAGING OF PROBABILITY MEASURES
}

\author{
Sun Man Chang
}

\begin{abstract}
Let $M$ be a compact space, and $X$ a complete sparable metric space. Let $P(X)$ denote the probability measures on $X$. Let $\lambda$ be a probability measure on $M$. Define a function $\varphi_{\lambda}$ from $C(M, P(X))$ to $P(X)$ by $\varphi_{\lambda}(T)(f)=\int T(t)(f) d \lambda(t)$ for every $T \in C(M, P(X)), f \in C(X)$. We show that $\varphi_{\lambda}$ is an open mapping.
\end{abstract}

1. Introduction. By a measure on a space $X$, we mean a regular Borel measure on $X$. A nonnegative measure is called a probability measure if its total mass is 1 .

Let $M$ be a compact space, and let $X$ be a complete separable metric space. Let $P(X)$ denote the collection of all probability measures on $X$. Let $C(X)$ denote the set of all bounded continuous real-valued functions on $X$. Give $P(X)$ the weak topology as functionals on $C(X)$. Let $C(M, P(X))$ denote the set of all continuous functions from $M$ into $P(X)$. Give $C(M, P(X))$ the topology of uniform convergence. Let $\lambda$ be a fixed probability measure on $M$. For each $T \in C(M, P(X))$, define a functional $\varphi_{\lambda}(T)$ on $C(X)$ by

$$
\varphi_{\lambda}(T)(f)=\int T(t)(f) d \lambda(t) .
$$

By [3, p. 35 and p. 47], $\varphi_{\lambda}(T)$ may be considered as a measure in $P(X)$. Write $\varphi_{\lambda}(T)=\int T(t) d \lambda(t)$. Denote the mapping $T \rightarrow \varphi_{\lambda}(T)$ by $\varphi_{\lambda}$. Then $\varphi_{\lambda}$ is a continuous function from $C(M, P(X))$ into $P(X)$. This paper is to show that $\varphi_{\lambda}$ is an open mapping. This result contains a result due to Eifler [2, Theorem 2.4] as a special case when $M$ consists of two points.

For a metric space $X$, we write $x_{n} \rightarrow x$ if $\left(x_{n}\right)_{n=1}^{\infty}$ converges to $x$ in $X$.

Acknowledgment. The author wishes to thank Professor Robert M. Blumenthal for his suggestion of this problem and for his invaluable suggestion for the idea of the proof of (B) in Theorem 3.1.

2. Basic lemmas. We will use the following notation in Lemma 2.1: Let $X$ and $Y$ be complete separable metric spaces, and $\pi: Y \rightarrow X$ a continuous function. Then $\pi$ induces a mapping also denoted by $\pi$, from $P(Y)$ to $P(X)$ and defined by $\pi \mu(E)=\mu\left(\pi^{-1}(E)\right)$. 
Lemma 2.1. Let $X$ be a complete separable metric space. Then there exist a totally disconnected complete separable metric space $G$, a continuous function $\varphi: G \rightarrow X$, and a continuous function $\widetilde{\varphi}: P(X) \rightarrow$ $P(G)$ such that $\varphi \tilde{\varphi}(\mu)=\mu$ for all $\mu \in P(X)$. Moreover, $\widetilde{\varphi}$ is affine:

$$
\tilde{\varphi}(\alpha \mu+(1-a) \nu)=\alpha \tilde{\varphi}(\mu)+(1-a) \tilde{\varphi}(\nu)
$$

for every $0<a<1$, and measures $\mu, \nu \in P(X)$.

Proof. Such a space $G$ is constructed by using a sequence $\left(F_{n}\right)_{n=1}^{\infty}$ of partitions of unity on $X$ having the property that each $F_{n}$ is subordinate to a cover of diameter less than $1 / n$. The details of its construction can be found in [1].

Let $X$ be a totally disconnected complete separable metric space. Consider sets of the form

$$
\begin{aligned}
M_{\mu, \varepsilon}\left(G_{1}, \cdots, G_{n}\right)= & \left\{\nu \in P(X):\left|\nu\left(G_{i}\right)-\mu\left(G_{i}\right)\right|<\varepsilon\right. \\
& \text { for } i=1, \cdots, n\}
\end{aligned}
$$

where $\varepsilon>0, \mu \in P(X)$, and $G_{1}, G_{2}, \cdots, G_{n}$ are mutually disjoint, both open and closed subsets of $X$ such that $\bigcup_{i=1}^{n} G_{i}=X$.

LemMa 2.2. The collection of sets of the form $\left(^{*}\right)$ is a base for the topology on $P(X)$.

Proof. For any open subset $U$ of $X$, let

$$
N_{\mu, \varepsilon}(U)=\{\nu \in P(X): \nu(U)+\varepsilon>\mu(U)\} .
$$

Since sets of the form $N_{\mu, \epsilon}(U)$ is a sub-base for the topology on $P(X)$, it suffices to show that

$$
N_{\mu, \varepsilon}(U) \cap M_{\mu, \varepsilon}\left(G_{1}, \cdots, G_{n}\right)
$$

contains a set of the form (*). Let $V \subseteq U$ be a both open and closed subset of $X$ such that $\mu(V)+\varepsilon / 2>\mu(U)$. Then $N_{\mu, \varepsilon / 2}(V) \subseteq N_{\mu, \varepsilon}(U)$, and it is easy to check that

$$
\begin{aligned}
& M_{\mu, \varepsilon / 2 n}\left(G_{1} \cap V, \cdots, G_{n} \cap V, G_{1} \backslash V, \cdots, G_{n} \backslash V\right) \\
& \subseteq N_{\mu, \varepsilon / 2}(V) \cap M_{\mu, \varepsilon}\left(G_{1}, \cdots, G_{n}\right) .
\end{aligned}
$$

This completes the proof.

\section{Main result.}

THEOREM 3.1. Let $M$ be a compact space, and let $X$ be a complete separable metric space. Let $\lambda$ be a probability measure on $M$. Then the function $\varphi_{\lambda}: C(M, P(X)) \rightarrow P(X)$ defined by 


$$
\varphi_{\lambda}(T)=\int T(t) d \lambda(t)
$$

is open.

Proof. The proof will be accomplished in two steps: (A) We establish the result when $X$ is totally disconnected. (B) We use (A) to complete the proof.

(A) Let $X$ be a totally disconnected complete separable metric space. Let $T \in C(M, P(X))$, and let $\mathscr{C}_{T}$ be a neighborhood of $T$. It suffices to show that $\varphi_{\lambda}\left(\mathscr{L}_{T}\right)$ is a neighborhood of $\varphi_{\lambda}(T)$. By Lemma 2.2, we may take $\mathscr{C}_{T}$ to be a set of the form:

$$
\mathscr{U}_{T}=\left\{S \in C(M, P(X)): S\left(M_{\imath}\right) \subseteq \mathscr{Y}_{\imath}, \text { for } i=1, \cdots, m\right\}
$$

where for each $i, M_{i}$ is a compact subset of $M$, and $\mathscr{C}_{i}$ is a basic open subset of $P(X)$ of the form:

$$
\mathscr{\mathscr { N }}_{i}=\left\{\theta \in P(X):\left|\theta\left(G_{i j}\right)-\theta_{\imath}\left(G_{\imath j}\right)\right|<\varepsilon, \text { for } j=1, \cdots, n_{\imath}\right\}
$$

where $\theta_{\imath} \in P(X)$ and $\left\{G_{i j}: j=1, \cdots, n_{\imath}\right\}$ is an open cover for $X$ consisting of mutually disjoint open subsets of $X$.

Let $\mathscr{C}$ be the collection of all nonempty subsets $U$ of $X$ such that $U=G_{1 j_{1}} \cap G_{2 j_{2}} \cap \cdots \cap G_{m j_{m}}$. Write $\mathscr{C}=\left\{U_{1}, \cdots, U_{n}\right\}$. Then $\mathscr{C}$ is an open cover for $X$ and $U_{i} \cap U_{j}=\varnothing$ if $i \neq j$.

Since each $G_{i j}$ is both open and closed, we have

$$
\delta=\operatorname{Max}_{i j} \operatorname{Max}_{t \in M_{i}}\left|T(t)\left(G_{i j}\right)-\theta_{\imath}\left(G_{i j}\right)\right|<\varepsilon .
$$

Let $\varepsilon_{0}=\varepsilon-\delta>0$. One sees immediately that if $S \in C(M, P(X))$ is such that $\operatorname{Max}_{t \in M}\left|S(t)\left(G_{\imath j}\right)-T(t)\left(G_{\imath j}\right)\right|<\varepsilon_{0}$ for all $i, j$, then $S \in \mathscr{U}_{T}$.

Let $\mu=\int T(t) d \lambda(t)$, and $a_{\imath}=\mu\left(U_{\imath}\right), 1 \leqq i \leqq n$. Then $\sum a_{i}=1$ and we may assume that $a_{n}>0$. Let $N$ be an integer such that $N \cdot a_{i}>n^{2}$ whenever $a_{\imath}>0,1 \leqq i \leqq n$. Define

$$
\mathscr{V}=\left\{\nu \in P(X):\left|\nu\left(U_{i}\right)-a_{\imath}\right|<\varepsilon_{0} / 2 N \text { for } i=1, \cdots, n\right\} .
$$

It suffices to show that $\varphi_{\lambda}\left(\mathscr{U}_{T}\right) \supseteq \mathscr{Y}$.

Let $\nu \in \mathscr{Y}$. Then $\nu=\nu_{1}+\cdots+\nu_{n}$, where $\nu_{i}$ is a measure on $X$ defined as $\nu_{i}(A)=\nu\left(A \cap U_{\imath}\right)$. Let $b_{i}=\nu\left(U_{i}\right)$. Then $\left|a_{i}-b_{\imath}\right|<\varepsilon_{0} / 2 N$, and $b_{\imath}>0$ whenever $a_{i}>0$.

Now, go back to the function $T$. Let $f_{i}(t)=T(t)\left(U_{\imath}\right)$. Then all $f_{i}, i=1, \cdots, n$, are continuous functions on $M$, and $\int f_{i}(t) d \lambda(t)=a_{i}$. We will construct continuous functions $g_{1}, \cdots, g_{n}$ on $M$ such that (1) $\int g_{i}(t) d \lambda(t)=b_{i}$, 
(2) $\operatorname{Max}_{t \in M}\left|g_{i}(t)-f_{i}(t)\right|<\varepsilon_{0} / n$, and

(3) $0 \leqq g_{i}(t) \leqq 1$ and $\sum_{i=1}^{n} g_{i}(t)=1$ for all $t$. Given $i=1, \cdots, n-1$, define $g_{i}$ as follows:

(a) If $b_{i}=a_{i}$, let $g_{i}(t)=f_{i}(t)$ for all $t$.

(b) If $b_{i}>a_{i}$, set $\delta_{i}=b_{i}-a_{i}<\varepsilon_{0} / 2 N$. Let $g_{i}(t)=f_{i}(t)+\left(\delta_{i} / a_{n}\right) f_{n}(t)$. Then,

$$
\begin{aligned}
f_{i}(t) & \leqq g_{i}(t) \leqq f_{2}(t)+\left(\varepsilon_{0} / 2 N \cdot a_{n}\right) f_{n}(t) \\
& \leqq f_{i}(t)+\left(\varepsilon_{0} / 2 n^{2}\right) f_{n}(t) .
\end{aligned}
$$

(c) If $b_{i}<a_{i}$, set $\delta_{i}=a_{i}-b_{i}<\varepsilon_{0} / 2 N$. Since $a_{i}>0$, so that $b_{i}>0$. Define $h_{i}(t)=0$, if $f_{i}(t) \leqq \delta_{i} ; h_{i}(t)=f_{i}(t)-\delta_{i}$, otherwise. Then $b_{i} \leqq \int h_{i}(t) d \lambda(t) \leqq a_{\imath}$. Let $b_{i}^{\prime}=\int h_{i}(t) d \lambda(t)$ and $g_{i}(t)=\left(b_{\imath} / b_{\imath}^{\prime}\right) h_{\imath}(t)$. Then $g_{\imath}(t) \leqq f_{i}(t)$ and

$$
\begin{aligned}
f_{i}(t)-g_{i}(t) & \leqq \delta_{i}+h_{i}(t)\left(1-b_{i} / b_{\imath}^{\prime}\right) \\
& \leqq \delta_{\imath}+\varepsilon_{0} / 2 N \cdot a_{\imath}<\varepsilon_{0} / n^{2} .
\end{aligned}
$$

Thus for $i=1, \cdots, n-1,0 \leqq g_{i} \leqq 1, \int g_{i}(t) d \lambda(t)=b_{i}$, and

$$
\operatorname{Max}_{t \in M}\left|g_{i}(t)-f_{i}(t)\right|<\varepsilon_{0} / n^{2} .
$$

Moreover, $g_{2}(t) \leqq f_{i}(t)+\left(\varepsilon_{0} / 2 n^{2}\right) f_{n}(t)$. Hence, $g_{1}(t)+\cdots+g_{n-1}(t) \leqq 1$ for all $t$. Let $g_{n}(t)=1-g_{1}(t)-\cdots-g_{n-1}(t)$. Then the functions $g_{1}, \cdots, g_{n}$ are as required. This completes the construction.

Now let $I, J$ be subsets of $\{1,2, \cdots, n\}$ such that $I=\left\{i: b_{i}>0\right\}$, $J=\left\{j: b_{j}=0\right\}$. For each $j \in J$, pick a measure $\alpha_{j} \in P\left(U_{j}\right)$. Define a continuous function $S: M \rightarrow P(X)$ by $S(t)=\sum_{i \in I}\left(g_{i}(t) / b_{i}\right) \nu_{i}+\sum_{j \in J} g_{j}(t) \alpha_{j}$. Clearly,

$$
\begin{aligned}
\varphi_{\lambda}(S) & =\sum_{i \in I}\left(\int \frac{g_{i}(t)}{b_{i}} d \lambda(t)\right) \nu_{i}+\sum_{j \in J}\left(\int g_{j}(t) d \lambda(t)\right) \alpha_{j} \\
& =\sum_{i \in I} \nu_{i}=\nu, \text { and } \operatorname{Max}_{t \in M}\left|S(t)\left(U_{i}\right)-T(t)\left(U_{i}\right)\right|<\varepsilon_{0} / n
\end{aligned}
$$

for all $i$. Since each $G_{i j}$ is a disjoint union of $U_{k}$, it follows that $\operatorname{Max}_{t \in M}\left|S(t)\left(G_{i j}\right)-T(t)\left(G_{i j}\right)\right|<\varepsilon_{0}$. Therefore, $S \in \mathscr{U}_{T}$. This completes the proof of $(\mathrm{A})$.

(B) Let $X$ be a complete separable metric space. To show that the mapping $\varphi_{\lambda}$ is open, it is equivalent to show the following: Let $T \in C(M, P(X))$, and $\mu=\varphi_{\lambda}(T)$. Let $\mu_{n}$ be a sequence converging to $\mu$ in $P(X)$. Then there is a sequence $T_{n} \rightarrow T$ in $C(M, P(X))$ such that $\varphi_{\lambda}\left(T_{n}\right)=\mu_{n}$.

For this purpose, we use Lemma 2.1 to pick a totally disconnected space $G$, continuous functions $\varphi: G \rightarrow X$ and $\widetilde{\varphi}: P(X) \rightarrow P(G)$, such 
that $\varphi \tilde{\varphi}(\mu)=\mu$, and that $\tilde{\varphi}$ is affine. Let $\tilde{\mu}_{n}=\widetilde{\varphi} \mu_{n}, \tilde{\mu}=\tilde{\varphi} \mu$. Then $\tilde{\mu}_{n} \rightarrow \tilde{\mu}$ in $P(G)$. Let $\widetilde{T}(t)=\tilde{\varphi} T(t)$ for each $t$. Then $\widetilde{T} \in C(M, P(G))$. It is easy to check that $\varphi_{\lambda}(\widetilde{T})=\widetilde{\varphi} \varphi_{\lambda}(T)$. In fact, this is obvious if there is a finite subset $\left\{t_{1}, \cdots, t_{n}\right\} \subseteq M$ with $\lambda\left\{t_{1}, \cdots, t_{n}\right\}=1$. In general, we may pick a net $\lambda_{\alpha} \rightarrow \lambda$ in $P(M)$ such that for each $\alpha$, $\lambda_{\alpha}\left(F_{\alpha}\right)=1$ for some finite subset $F_{\alpha}$ of $M$. Thus, $\varphi_{\lambda_{\alpha}}(\widetilde{T})=\widetilde{\varphi} \varphi_{\lambda_{\alpha}}(T)$. Let $\alpha \rightarrow \infty$, then we obtain

$$
\varphi_{\lambda}(\widetilde{T})=\widetilde{\varphi} \varphi_{\lambda}(T) .
$$

Hence $\varphi_{\lambda}(\widetilde{T})=\tilde{\mu}$. Since by (A), the function

$$
\varphi_{\lambda}: C(M, P(G)) \longrightarrow P(G)
$$

is open, hence, we may pick $\widetilde{T}_{n} \rightarrow \widetilde{T}$ in $C(M, P(G))$ such that $\varphi_{\lambda}\left(\widetilde{T}_{n}\right)=$ $\tilde{\mu}_{n}$. Let $T_{n}(t)=\varphi \widetilde{T}_{n}(t)$. Then $T_{n} \rightarrow \varphi \widetilde{T}=T$ in $C(M, P(X))$, and the same argument in proving $\varphi_{\lambda}(\widetilde{T})=\widetilde{\varphi} \varphi_{\lambda}(T)$ will give $\varphi_{\lambda}\left(T_{n}\right)=\varphi \varphi_{\lambda}\left(\widetilde{T}_{n}\right)$. Therefore,

$$
\varphi_{\lambda}\left(T_{n}\right)=\varphi \tilde{\mu}_{n}=\mu_{n} .
$$

This proves (B), and so completes the proof of this theorem.

As a special case of Theorem 3.1, we let $M=\{1,2\}$ with the discrete topology. We obtain Eifler's result [2]:

Corollary 3.2. Let $X$ be a complete separable metric space, and let $0<\lambda<1$. Then the function

$$
\lambda: P(X) \times P(X) \longrightarrow P(X)
$$

defined by $(\mu, \nu) \rightarrow \lambda \mu+(1-\lambda) \nu$ is open.

\section{REFERENCES}

1. R. M. Blumenthal and H. H. Corson, On continuous collections of measures, Ann. Inst. Fourier, Grenoble, 20 (1970), 193-199.

2. L. Q. Eifler, Open mapping theorem for probability measures on metric spaces, (to appear).

3. K. R. Parthasarathy, Probability Measures on Metric Spaces, Academic Press (1967).

Received October 21, 1975.

UNIVERSITY OF WASHINGTON 



\section{PACIFIC JOURNAL OF MATHEMATICS}

\section{EDITORS}

RICHARD ARENS (Managing Editor) University of California

Los Angeles, California 90024

R. A. Beaumont

University of Washington

Seattle, Washington 98105
J. DUGUNDJI

Department of Mathematics University of Southern Californı

Los Angeles, California 90007

D. Gilbarg and J. Milgram

Stanford University

Stanford, California 94305

\section{ASSOCIATE EDITORS}
E. F. BECKENBACH
B. H. NeumanN
F. WOLF
K. YosHIDA

\section{SUPPORTING INSTITUTIONS}

UNIVERSITY OF BRITISH COLUMBIA

CALIFORNIA INSTITUTE OF TECHNOLOGY

UNIVERSITY OF CALIFORNIA

MONTANA STATE UNIVERSITY

UNIVERSITY OF NEVADA

NEW MEXICO STATE UNIVERSITY

OREGON STATE UNIVERSITY

UNIVERSITY OF OREGON

OSAKA UNIVERSITY
UNIVERSITY OF SOUTHERN CALIFORNIA

STANFORD UNIVERSITY

UNIVERSITY OF TOKYO

UNIVERSITY OF UTAH

WASHINGTON STATE UNIVERSITY

UNIVERSITY OF WASHINGTON

* * * *

AMERICAN MATHEMATICAL SOCIETY

NAVAL WEAPONS CENTER 


\section{Pacific Journal of Mathematics \\ Vol. 65, No. $1 \quad$ September, 1976}

David Lee Armacost, Compactly cogenerated LCA groups ............. 1

Sun Man Chang, On continuous image averaging of probability measures ...... 13

J. Chidambaraswamy, Generalized Dedekind $\psi$-functions with respect to a

polynomial. II................................... 19

Freddy Delbaen, The Dunford-Pettis property for certain uniform algebras ..... 29

Robert Benjamin Feinberg, Faithful distributive modules over incidence

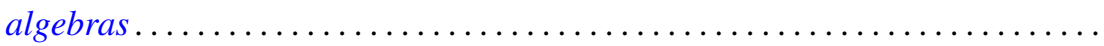

Paul Froeschl, Chained rings . . . . . . . . . . . . . . . . . . . .

John Brady Garnett and Anthony G. O'Farrell, Sobolev approximation by a sum

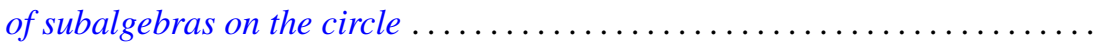

Hugh M. Hilden, José M. Montesinos and Thomas Lusk Thickstun, Closed

oriented 3-manifolds as 3-fold branched coverings of $S^{3}$ of special type.....

Atsushi Inoue, On a class of unbounded operator algebras ................

Peter Kleinschmidt, On facets with non-arbitrary shapes.

Narendrakumar Ramanlal Ladhawala, Absolute summability of Walsh-Fourier

series

Howard Wilson Lambert, Links which are unknottable by maps . . . . . . . . . . .

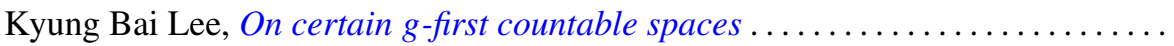

Richard Ira Loebl, A Hahn decomposition for linear maps .................

Moshe Marcus and Victor Julius Mizel, A characterization of functionals on $W_{1}^{p}$ possessing autonomous kernels. I . .

James Miller, Subordinating factor sequences and convex functions of several

variables.

Keith Pierce, Amalgamated sums of abelian l-groups ...

Jonathan Rosenberg, The $C^{*}$-algebras of some real and $p$-adic solvable

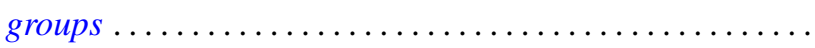

Hugo Rossi and Michele Vergne, Group representations on Hilbert spaces defined

in terms of $\partial_{b}$-cohomology on the Silov boundary of a Siegel domain . .

Mary Elizabeth Schaps, Nonsingular deformations of a determinantal

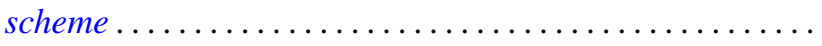

S. R. Singh, Some convergence properties of the Bubnov-Galerkin method...

Peggy Strait, Level crossing probabilities for a multi-parameter Brownian

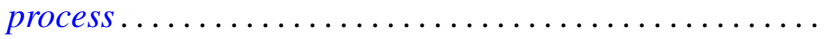

Robert M. Tardiff, Topologies for probabilistic metric spaces .

Benjamin Baxter Wells, Jr., Rearrangements of functions on the ring of integers of

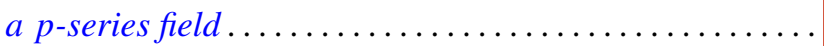

Robert Francis Wheeler, Well-behaved and totally bounded approximate identities for $C_{0}(X)$.

Delores Arletta Williams, Gauss sums and integral quadratic forms over local

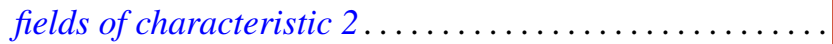

John Yuan, On the construction of one-parameter semigroups in topological 